\title{
Pretreatment with yeast derived complex dietary-polysaccharide leads to suppressed gut inflammation, altered microbiota composition and increased immune regulatory short-chain fatty acid production in C57BL/6 mice
}

Radhika Gudi $^{1 *}$, Jada Suber ${ }^{*}$, Robert Brown ${ }^{1 *}$, Benjamin M. Johnson ${ }^{1 *}$, and Chenthamarakshan $\operatorname{Vasu}^{1,5}$

${ }^{1}$ Department of Microbiology and Immunology, College of Medicine, Medical University of South Carolina, Charleston, SC-29425

Running title: Dietary polysaccharide on gut inflammation

*contributed equally

${ }^{5}$ Address Correspondence: Chenthamarakshan Vasu

Microbiology and Immunology

Medical University of South Carolina

173 Ashley Avenue, BSB208E

Charleston, SC-29425

Phone: 843-792-1032, Fax: 843-792-9588

Email: vasu@musc.edu

Sources of Support: Medical University of South Carolina internal funds; National Institutes of Health grants R21AI133798, R21AI133798 ODS administrative supplement, R21AI133798 and R21AI136339-02S1.

Conflict of Interest: Authors do not have any conflict(s) of interest to disclose. 


\section{ABSTRACT}

2 Background: $\beta$-Glucans (BGs), a group of complex non-digestible polysaccharides, are

3 considered to have beneficial health effects due to their immune modulatory properties and are

4 considered as dietary supplements. However, the impact of oral administration of high-pure, well-

5 defined BGs on gut inflammation, and the influence of intestinal microbiota and short-chain fatty

6 acid (SCFA) on the therapeutic effect are largely unknown.

7 Objectives: The aim of this study, using a mouse model of chemical induced colitis, was to

8 investigate the impact of oral administration of high-pure yeast BG (YBG) on the susceptibility to

9 colitis, gut immune function, and structure and function of microbiota.

10 Methods: To determine the impact of oral administration of YBG on colitis susceptibility, eight

11 week old C57BL/6 (B6) mice were pre-treated with YBG (250 $\mu \mathrm{g} / \mathrm{mouse} /$ day) and given dextran

12 sulfate sodium (DSS) in drinking water $(2.5 \% \mathrm{w} / \mathrm{v})$ and examined for the symptoms and features

13 of colitis. To assess the effect of oral administration of YBG on gut mucosa and microbiota

14 structure and function, and gut immune regulation, we determined the microbiota composition,

15 fecal SCFA levels, and intestinal $\mathrm{T}$ cell phenotype and cytokine secretion. The role of gut

16 microbiota in YBG treatment induced modulation of gut inflammation and immune function were

17 determined in B6 mice treated with broad-spectrum antibiotic cocktail $(1 \mathrm{~g} / \mathrm{L}$ ampicillin, $0.5 \mathrm{~g} / \mathrm{L}$

18 vancomycin, $1 \mathrm{~g} / \mathrm{L}$ neomycin, and $1 \mathrm{~g} / \mathrm{L}$ metronidazole) in drinking water.

19 Results Compared to untreated mice, B6 mice that received prolonged pre-treatment with YBG

20 showed diminished severity of different features of DSS-induced colitis including overall loss of

21 body weight $(P<0.001)$, shortening of colon $(P=0.016)$ and histopathology $(P=0.01)$. However,

22 high-pure YBG has no beneficial effect in terms of suppressing colitis severity when consumed

23 only during the disease stage. Compared to untreated controls, YBG pre-treated mice showed 
24 higher regulatory $\mathrm{T}$ cell (Treg) frequencies $(P=0.043)$ in the gut mucosa, a shift in the abundance

25 of gut microbiota towards polysaccharide-fermenting bacterial phyla Bacteroides $(P=0.049)$ and

26 Verrucomicrobia (Mean \pm SD: control $=13.0 \pm 0.33$ vs $Y B G=10.9 .7 \pm 0.69$ ) and diminished

27 Firmicutes $(P<0.001)$ and Proteobacteria $(P<0.001)$, and significantly higher production of SCFA

28 such as acetic acid $(P=0.016)$, propionic acid $(P=0.026)$ and butyric acid $(P=0.013)$. Depletion of

29 gut microbiota in YBG-fed B6 mice using broad spectrum antibiotics caused not only elimination

30 of YBG treatment associated SCFA production and Treg increase, but also profound aggravation

31 of the pathological features of colitis such as loss of body weight $(P<0.01)$ and colonic

32 inflammation $(P=0.04)$ compared to that of $Y B G$ treated control mice.

33 Conclusions: Oral consumption of high-pure BG promotes a healthy gut homeostasis and immune

34 regulation, and minimizes susceptibility to DSS induced colitis in B6 mice in a microbiota (and

35 microbial SCFA) - dependent manner. On the contrary, YBG consumption when gut mucosa and

36 microbiota are compromised not only reverses this protection but also increases the susceptibility

37 to gut inflammation and disease severity, perhaps through its direct interaction with gut immune

38 cells. In conclusion, while YBG consumption may be beneficial for gut health and to prevent gut

39 inflammation in healthy individuals and under intact microbiota, this immune stimulatory dietary

40 supplement may not have any health benefits in individuals with active gut inflammation and could

41 cause adverse effect in those who are on oral antibiotics.

43 Key words: Yeast $\beta$-glucan; gut mucosa, Gut microbiota; short-chain fatty acid, colitis, gut

44 inflammation, immune modulation; immune regulation. 


\section{INTRODUCTION}

47 Dietary approaches have increasingly been considered for modulating immune function, and

48 preventing autoimmunity and other clinical conditions. $\beta$-glucans (BGs) are non-digestible

49 complex dietary polysaccharides (CDPs) of varying structures ( $\beta$-1,3-D-glucan, $\beta$-1,3/1,6-D-

50 glucan, $\beta$-1,3/1,4-D-glucan and $\beta$-1,4-D-glucan) commonly found in plants, cereals, bacteria,

51 yeast, and fungi (1). While BGs can potentiate the function of immune system directly $(2,3)$, BG-

52 containing preparations have been promoted also as daily dietary supplements to enhance

53 metabolic functions, and as prebiotics (4). On the other hand, clinical studies using YBG

54 preparations reported conflicting outcomes in terms of their potential benefits as dietary

55 supplements (5-9). Studies using animal models to determine the potential of BG preparations and

56 BG containing diets to modulate gut inflammation have also produced conflicting outcomes (10-

57 15). Furthermore, inconsistent results on colitis susceptibility of Dectin-1 (BG interacting receptor)

58 knockout mice have been reported $(16,17)$.

59 Differences observed in the impact of BG dietary supplements on host health could be due multiple

60 factors including 1) the purity and/or the structure of the BG employed, 2) degree of direct

61 interactions of those BG preparations with host receptors, 3) possible association between gut

62 microbes and BG induced effects in the host, and differences in the structure and function of basal

63 gut microbiota. Determination of the true effect of oral consumption of BGs on the host, thus,

64 requires systematic pre-clinical studies using well-defined high-pure BGs. Further, studying the

65 direct immune modulatory effect of BGs on gut mucosa and the indirect, microbiota-dependent,

66 effect is necessary to better understand mechanisms of immune modulation induced these dietary

67 complex polysaccharides. Such studies will also help explain the contradictory effects of these

68 agents on the host. For this study, we hypothesized that oral administration of a highly purified

69 BG [ $\beta$-1,3-linked D-glucose molecules ( $\beta$-1,3-D-glucan) with $\beta$-1,6-linked side chains] from 
Baker's yeast (Saccharomyces cerevisiae) (YBG) prevents autoimmunity $(18,19)$ can enhance

71 immune modulation by directly interacting with the gut mucosa and by serving as a fermentation

72 substrate for gut commensals that produce immune regulatory metabolites. Therefore, we

73 examined the impact of oral administration of high pure YBG on gut inflammation employing

74 DSS induced colitis in C57BL/6 (B6) mice as the model, and assessed the contribution of gut

75 microbiota to YBG consumption associated enhanced gut immune regulation and suppression of

76 colitis susceptibility.

Materials and Methods

Mice

C57BL/6 (B6) mice were originally purchased from the Jackson laboratory. Foxp3-GFP-knockin

81 (Foxp3-GFP) mice in B6 background were provided by Dr. Vijay Kuchroo (Harvard Medical

82 School, MA). Breeding colonies of these strains were established and maintained in the pathogen-

83 free facility of Medical University of South Carolina (MUSC). Mice were given autoclaved rodent

84 diet (20), which contains 18.6\% (wt:wt) protein, 6.2\% (wt:wt) fat, and 44.2\% (wt:wt)

85 carbohydrates (Harlan Laboratories, Teklad Global 18\% Protein Rodent diet no. 2018), and

86 autoclaved water ad libitum. All animal studies were approved by the animal care and use

87 committee at MUSC. Since B6 mice are highly susceptible to DSS induced distal gut inflammation

88 and has been widely used as a model of ulcerative colitis (UC) (21-24), we employed this strain

89 for determining the impact of YBG on DSS induced colitis. While most experiments described in

90 this study used age matched females for control and test groups, our initial experiments showed

91 YBG treatment induced similar protection of male B6 mice from colitis. However, since mortality

92 was as high as $50 \%$ by day 10 with control males with DSS colitis, to be able to compare similar 
93 number of controls and YBG treated mice side-by-side, we preferred females for this study. B6-

94 Foxp3-GFP report mice that express GFP under Foxp3 promoter and only in regulatory T cell were

95 used in some experiments to visualize and enumerate Tregs by FACS unambiguously.

\section{YBG and other reagents}

97 Purified YBG (glucan from Baker's yeast, S. cerevisiae), $\geq 98 \%$ pure, was purchased from Sigma-

98 Aldrich and the purification method has been described before (18). This agent was tested for

99 specific activity using thioglycolate-activated macrophages as described before by us and others

100 (25-27). PMA, ionomycin, Brefeldin A, and purified and conjugated antibodies were purchased

101 from Sigma-Aldrich, BD Biosciences, eBioscience, Invitrogen, R\&D Systems, and Biolegend

102 Laboratories. In some experiments, intestinal epithelial cells were depleted by Percoll gradient

103 centrifugation (28) or using A33 antibody (Santa Cruz Biotechnology) and anti-rabbit IgG-

104 magnetic beads $(29,30)$. Oligonucleotide probes for qPCR were custom synthesized by Thermo

105 Fisher. Magnetic bead-based multiplex assay kits were purchased from Invitrogen. Additional

106 details of commercial products used in this study are listed in supplemental table 1.

\section{$107 \quad$ YBG treatment}

108 Eight week old B6 mice or B6-Foxp3-GFP mice were given YBG suspension in saline (250

$109 \mu \mathrm{g} / \mathrm{mouse} /$ day) for up to 30 days by oral gavage. Mice that were subjected to colitis induction using

110 DSS, as described below, continued to receive YBG or saline until the mice were euthanized on

111 day 40. In some experiments, YBG and saline treatments were initiated only 1 day before initiating

112 the DSS treatment to determine the effect of treatment initiation at disease stage. This dose of YBG

113 was considered because it is a human relevant dose of BG dietary supplements (500 to 1000

$114 \mathrm{mg} /$ day) equaling approximately $10 \mathrm{mg} / \mathrm{kg}$ body weight. Further, as mentioned in our previous

115 report (29), lower doses or short-term treatments do not produce observable immune modulation 
116 in the gut mucosa. We also found that even a very short-term (3 day) treatment with higher doses

117 such as $2000 \mu \mathrm{g} /$ mouse/day produce pro-inflammatory response in the gut mucosa in Dectin-1

118 dependent manner (29); hence a relatively lower daily dose was considered for this study.

\section{Induction of DSS colitis and disease evaluation}

120 To induce colitis, mice were maintained on drinking water containing $2.5 \%(\mathrm{w} / \mathrm{v})$ DSS salt

121 (36,000-50,000 MW) for 5 consecutive days. After 5 days, mice were switched to regular water

122 until euthanasia on day 10, post-DSS treatment initiation. Mice were weighed, and examined for

123 the stool consistency and presence of occult blood every day, and the severities were scored as

124 described before (31). In some experiments, mice were given a cocktail of antibiotics starting 5

125 days before DSS treatment. Mice were euthanized 10 days post-DSS treatment initiation, intestinal

126 tissues were collected, the colon lengths were measured, and distal colon tissues were subjected to

127 histochemical staining to determine the severity of inflammation. Distal colon tissues were fixed

128 in $10 \%$ formaldehyde, 5 - $\mu \mathrm{m}$ paraffin sections were cut, and stained with hematoxylin and eosin.

129 Stained sections were analyzed using the 0-6 grading criteria described before (32) for

130 inflammatory cell infiltrate, epithelial changes and mucosal architecture, and the values were

131 averaged to calculate the overall inflammation score of individual mice.

\section{Immunological assays and qPCR}

133 Cells from the small intestinal lamina propria (SiLP), large intestinal lamina propria (LiLP) and 134 mesenteric lymph node (MLN) were examined for immune cell phenotype and/or cytokine profiles

135 by FACS and multiplex assay respectively as described in our recent reports $(29,30,33)$. Briefly:

136 for determining Treg frequencies, freshly isolated cells were stained for CD4 and Foxp3. For

137 determining cytokine positive T cells frequencies, cells were cultured in the presence or PMA,

138 Ionomycin and Brefeldin A for $4 \mathrm{~h}$, before staying for CD4 and intracellular cytokines. For 
139 determining secreted cytokine levels, cells were cultured in the presence of anti-CD3 antibody for

$14024 \mathrm{~h}$ and the spent media were subjected to multiplex assay and read using FlexMap 3D instrument

141 (Luminex). SYBR green based qPCR assay was run in a CFX96 Touch real time PCR machine

142 (BioRad) for determining cytokine gene expression levels in the RNA prepared from 143 cryopreserved intestinal tissues.

\section{$144 \quad 16 S$ rRNA gene targeted sequencing and bacterial community profiling}

145 Total DNA was prepared from the fecal pellets for 16S rRNA gene targeted sequencing and

146 bacterial community profiling as detailed in our previous reports $(19,34,35)$ with minor

147 modifications. Briefly, the sequencing reads were fed into the Metagenomics application of

148 BaseSpace (Illumina) for performing taxonomic classification of 16S rRNA targeted amplicon

149 reads using an Illumina-curated version of the GreenGenes taxonomic database which provided

150 raw classification output at multiple taxonomic levels. The sequences were also fed into QIIME

151 open reference operational taxonomic units (OTU) picking pipeline (36) using pre-processing

152 application of BaseSpace. The OTUs were compiled to different taxonomical levels based upon

153 the percentage identity to GreenGenes reference sequences (i.e. $>97 \%$ identity) and the percentage

154 values of sequences within each sample that map to respective taxonomical levels were calculated.

155 The OTUs were also normalized and used for metagenomes prediction of Kyoto Encyclopedia of

156 Genes and Genomes (KEGG) orthologs employing PICRUSt as described before (37-40). The

157 predictions were summarized to multiple levels and functional categories were compared among

158 control and YBG fed groups using the statistical Analysis of Metagenomic Profile Package

159 (STAMP) as described before (38, 41). STAMP and web-based MicrobiomeAnalyst (42)

160 applications were also employed for visualization and/or statistical analysis of microbiome data.

\section{Depletion of gut microbiota}


162 Mice were given a broad-spectrum antibiotic cocktail (ampicillin (1 g/l), vancomycin $(0.5 \mathrm{~g} / 1)$,

163 neomycin $(1 \mathrm{~g} / \mathrm{l})$, and metronidazole $(1 \mathrm{~g} / \mathrm{l})$-containing drinking water during the last 5 days of

164 YBG treatment to deplete gut microbiota as described in our recent report (29). Fecal pellet

165 suspensions were cultured under aerobic and anaerobic conditions to assess microbiota depletion

166 (29). In some experiments, antibiotic treatment was continued during colitis induction using DSS.

\section{Determination of fecal SCFA levels}

168 Fresh fecal samples were collected from individual mice, stored at $-80^{\circ} \mathrm{C}$, and shipped on dry ice

169 to Microbiome Insights (Vancouver, Canada) for SCFA analysis. SCFA were detected by this

170 service provider using gas chromatography (Thermo Trace 1310), coupled to a flame ionization

171 detector, using Thermo TG-WAXMS A GC Column by following a previously described method

$172(43)$.

\section{Statistical analysis}

174 Mean, SD, and statistical significance $(P$-value $)$ were calculated, graphic visualizations were made

175 using Excel (Microsoft), Prism (GraphPad), Morpheus (Broad Institute), STAMP (38, 41) and/or

176 MicrobiomeAnalyst (42) applications. Briefly, unpaired, two-tailed $t$-test or two-tailed non-

177 parametric Mann-Whitney test was employed for most assays where test (YBG fed or microbiota

178 depleted) group samples were compared to that of respective control (saline fed or those with intact

179 microbiota) group, unless specified here. For DSS colitis associated weight loss, stool consistency

180 and stool levels of blood, test group mean of each time point was compared to the respective time-

181 point control group mean by unpaired, two-tailed $t$-test. To compare the number of mice with grade

$182 \leq 3$ and $\geq 4$ colon histopathology scores in test and control groups, two-tailed Fishers' exact

183 contingency test was employed. Log-rank test was employed for comparing the survival rates of

184 YBG treated mice with that of controls. Statistical analysis of microbiota at genus level as well as 
185 the number of sequences representing (predicted) specific metabolic pathways were done in

186 STAMP employing two-sided Welch's $t$-test and FDR corrected using Benjamini and Hochberg

187 approach, and the heatmap visualization was done in Morpheus. MicrobiomeAnalyst was

188 employed for generating principal component (PC) analysis representing $\beta$-diversity (Bray Curtis

189 distance) and $\alpha$-diversity / species richness / Chaol estimator plots, and for statistical significances

190 by permutational multivariate analysis of variance and Mann-Whitney approaches respectively. A

$191 p$-value of $\leq 0.05$ was considered significant.

192 Results

193 Prolonged pretreatment with YBG diminishes susceptibility to DSS induced colitis in B6

194 mice.

195 Eight week old female B6 mice were orally administered with YBG and subjected to colitis

196 induction using DSS as shown in Fig. 1A, and examined for gut inflammation associated features

197 to determine the impact of pre-treatment with YBG on colitis susceptibility. As observed in Fig.

198 1B, YBG treated mice were relatively less susceptible to DSS colitis associated weight loss

199 compared to control mice. Significant difference in the body weight between control and YBG

200 treated groups was observed starting day $6(P=0.027)$ and peaked at day $10(P<0.001)$. As shown

201 in Fig. 1C, YBG treated mice also showed significant resistance to colitis associated shortening

202 of the colon compared to that of control mice $(P=0.016)$. Overall DSS induced histological injury

203 scores of YBG treated mice was significantly lower $(P=0.016)$ as compared to that of control group

204 (Fig. 1D). The colon of DSS-treated control mice exhibited more severe inflammatory infiltrates

205 and ulceration compared to YBG treated mice. Further, compared to DSS treated control mice,

206 better stool consistency (supplemental Fig. 1A) and lesser fecal blood (supplemental Fig. 1B)

207 were detected in YBG-fed mice that were treated with DSS at the time-point in which these 
208 features were most severe. Of note, prolonged pre-treatment of male B6 mice with YBG produced

209 similar protection to that of B6 female counterparts, but control group showed 50\% mortality prior

210 to termination of the experiment (not shown). Further, we also observed that mice that were given

211 YBG only during colitis induction did not show any difference in the severity of colitis associated

212 parameters compared to control mice (Supplemental Fig. 2).

\section{YBG treated and control B6 mice with colitis showed distinct immune characteristics}

214 Since B6 mice that were pretreated with YBG showed less severe colon inflammation, T cells

215 from their gut associated mesenteric lymph nodes (MLNs) were examined for phenotypic

216 properties. As shown in Fig. 2A and supplemental Fig. 3, Foxp3 $+\mathrm{T}$ regulatory cell (Treg)

217 frequencies were significantly higher in the MLN of YBG-fed mice $(P=0.0043)$ compared to

218 control mice. FACS analyses revealed significantly higher frequencies of IL10+ $(P=0.0043)$, IL4+

$219(P=0.041)$ and IL9+ $(P=0.015)$ and lower IFN $\gamma+(P=0.0087), \operatorname{IL} 17+(P=0.0022)$ and IL22+

$220(P=0.0152) \mathrm{T}$ cell frequencies in YBG-fed mice compared to controls. To validate these

221 observations, the levels of cytokines secreted by MLN cell were determined. Multiplex cytokine

222 assay revealed significantly higher secretion of IL10+ $(P=0.0087)$, IL4+ $(P=0.0152)$ and IL9+

$223(P=0.041)$ and lower IFN $\gamma+(P=0.0087), \operatorname{IL} 17+(P=0.041)$ and IL22 $+(P=0.026)$ by MLN cells

224 from YBG fed mice, compared to that of control mice, upon ex vivo activation using anti-CD3

225 antibody (Fig. 2B).

Prolonged YBG treatment results in changes in the fecal microbiota composition in B6 mice

227 Since only prolonged with YBG prior to DSS treatment reduced the susceptibility to colitis in B6

228 mice, we determined if YBG treatment alters gut microbiota structure and function in them.

229 Compilation of OTU data generated from 16S rRNA sequences of fecal samples to different

230 taxonomical levels showed that, compared to the pre-treatment time-point, abundances of major 
231 phyla changed significantly in YBG-fed mice, but not in control mice (supplemental Fig. 4A).

232 YBG treatment resulted in increase in the abundances of Bacteroidetes $(P=0.049)$, and

233 Verrucomicrobia (Mean \pm SD: control $=13.0 \pm 0.33$ vs $Y B G=10.9 .7 \pm 0.69$ ) phyla members and

234 decrease in Firmicutes $(P<0.001)$ and Proteobacteria $(P<0.001)$ in their fecal samples.

235 Interestingly, at genus level, abundances of many major microbial communities are altered

236 significantly after YBG treatment (Fig. 3A). Compared to pre-treatment time-point, significant

237 reductions in the abundance of microbial communities belonging to multiple genera including

238 Ruminococcus (phylum: Firmicutes; $P=0.003$ ), Lactobacillus (phylum: Firmicutes; $P=0.02$ ),

239 Oscillospira (phylum: Firmicutes; $P<0.001$ ), Dehalobacterium (phylum: Firmicutes; $P<0.006$ ),

240 and Odoribacter (phylum: Firmicutes; $P<0.001$ ) were observed after YBG treatment. On the other

241 hand, abundance of microbial communities belonging to many genera including

242 Parabacteroidetes (phylum: Bacteroidetes; $P<0.005$ ), Prevotella (phylum: Bacteroidetes;

$243 P<0.002$ ), Sutterella (phylum: Proteobacteria; $P<0.007$ ), and Bifidobacterium (phylum:

244 Actinobacteria; $P=0.048$ ) were increased after YBG treatment. Interestingly fecal samples

245 collected after YBG treatment showed lower gut microbial $\alpha$-diversity / species richness

$246(P<0.001)$ compared to pretreatment time-point (Supplemental Fig. 4B). Further, $\beta$-diversity (Bray

247 Curtis distance) analysis of fecal microbiota showed significant distance $(P=0.007)$ in the

248 clustering / separation of samples collected before and after YBG treatment (Supplemental Fig.

249 4C). As shown in Fig. 3C, OTU-based prediction of metabolic functions of gut microbes revealed

250 overrepresentation of many pathways including that of carbohydrate metabolism $(P=0.031)$,

251 glycan biosynthesis and metabolism $(P=0.027)$ and biosynthesis of secondary metabolites

$252(P=0.01)$ in B6 mouse gut microbiota after YBG treatment compared to pre-treatment time-point. 
254 mice

255 Since YBG treatment altered gut microbiota and suppressed colitis susceptibility in B6 mice, we 256 determined if YBG treatment alone has an impact on gut immune phenotype. Supplemental fig. 5

257 shows that the primarily small intestine (ileum), but not large intestine (colon), of mice that 258 received YBG for 30 consecutive days expressed significantly higher levels of pro-inflammatory 259 cytokine TNF $\alpha(P=0.0039)$ and immune regulatory enzyme Raldh1A2 $(P=0.001)$ compared to 260 that of untreated counterparts. On the other hand, significantly higher levels of IL10 expression 261 was detected in both ileum $(P=0.0017)$ and colon $(P=0.0042)$ of YBG treated mice compared to

262 controls. We then determined the T phenotype of gut mucosa in mice that received YBG for 30 263 consecutive days by assessing the Foxp3 + T cell frequencies and cytokine production. As shown 264 in supplemental Fig. 6A and Fig. 4A, significantly higher frequencies of Foxp3+ T cells were 265 detected in mesenteric LNs (MLNs) $(P=0.016)$, small intestine lamina propria (SiLP) $(P=0.0079)$ 266 and large intestinal lamina propria (LiLP) $(P=0.0159)$ of YBG treated mice compared to controls.

267 Further, compared to that of controls, small intestinal cells from YBG treated mice produced 268 significantly higher amounts of IL10 $(P=0.016)$ and IL17 $(P=0.0044)$. On the other hand, colonic 269 immune cells of YBG treated mice produced higher amounts of IL10 $(P=0.0079)$, but not IL17 or $270 \quad$ IFN $\gamma$.

271 We then determined the influence of microbiota on YBG consumption-associated immune 272 modulation in B6 mice after depleting gut bacteria using broad-spectrum antibiotics (Supplemental 273 fig. 7). Depletion of gut bacteria alone caused reduction in Foxp3 $+\mathrm{T}$ cell frequencies in both 274 control and YBG recipient mice (supplemental Fig. 6B and Fig. 4B), relative to their counterparts 275 with intact microbiota (shown in Fig. 4A). Further, SiLP cells, but not LiLP cells, from microbiota- 
276 depleted YBG recipient mice showed relatively higher frequencies of Foxp3+ T cells $(P=0.0041)$

277 compared to their control counterparts. On the other hand, small intestine of antibiotic-treated

278 YBG-recipients showed higher IL10 $(P=0.0087)$, IL17 $(P=0.0159)$ and IFN $\gamma(P=0.041)$

279 production, compared to microbiota-depleted controls. Immune cells from large intestine of YBG-

280 treated microbiota-depleted mice produced significantly higher amounts of IL17 $(P=0.0034)$ and

$281 \operatorname{IFN} \gamma(P=0.0039)$, but not IL10, compared to control counterparts.

YBG treatment results in increased immune regulatory SCFA production in B6 mice

283 Since fermentation of non-digestible sugars by colonic bacteria can result in the generation of

284 metabolites including SCFA, fecal SCFA levels in control and YBG treated B6 mice with intact 285 and depleted gut microbiota were determined. As observed in Fig. 5A, significantly higher fecal 286 acetic acid $(P=0.016)$, propionic acid $(P=0.026)$ and butyric acid $(P=0.013)$, and lower valeric 287 acid $(P=0.036)$ levels were detected in YBG-treated mice compared to their control counterparts.

288 On the other hand, depletion of gut microbiota not only resulted in the elimination of YBG289 treatment associated effect on SCFA production, but caused profound suppression of overall SCFA 290 levels in both control and YBG treated mice (Fig. 5B) compared to that of mice with intact 291 microbiota (Fig. 5A).

293 To determine if the YBG treatment associated protection of B6 mice from colitis is microbiota

294 dependent, YBG treated and control B6 mice were given broad spectrum antibiotics to deplete gut 295 microbiota and given DSS to induce colitis as depicted in Fig. 6A. As observed in Fig. 6B and C, 296 control and YBG treated mice with intact gut microbiota showed weight loss and disease severity 297 comparable to that of Fig. 1. Importantly, control mice with microbiota depletion showed relatively 
298 lesser weight loss and disease severity compared to their control counterparts, and the difference

299 was statistically significant $(P=0.012)$ only at 10 week time point (Fig. 6B; left panel). However,

300 colon inflammation scores were not statistically significant in these mice (Fig. 6C; left panel). On

301 the other hand, YBG-treated, microbiota-depleted mice showed significantly higher weight loss

302 and disease severity starting at day 6 time-point $(P=0.016)$ and more severe thereafter $(P<0.01)$

303 compared to untreated mice with intact microbiota. Further, YBG treated, microbiota depleted

304 mice showed higher colon inflammation $(P=0.047)$ compared to control mice (Fig. 6C; right

305 panel). Examination of cytokine profiles of immune cells from the colons revealed significantly

306 higher production of pro-inflammatory IFN $\gamma(P<0.001)$ and IL17 $(P<0.001)$, and comparable anti-

307 inflammatory IL10, production in YBG-treated, microbiota depleted mice compared to YBG

308 treated mice with intact gut microbiota (Fig. 6D). However, cells from microbiota depleted control

309 mice showed diminished IFN $\gamma$ production compared to cells from controls with intact microbiota

$310 \quad(P<0.001)$.

\section{Discussion}

312 Literature suggests that certain complex dietary polysaccharides such as BGs of different microbial

313 and plant origin affect the host immune function (44-48). While previous studies using various BG

314 preparations and BG rich diets have shown that they have the ability to suppress gut inflammation

315 in colitis models (10-14), others have shown that BGs aggravate the colitis severity (15). Further,

316 while it has been shown that genetic deletion of a BG interacting receptor, Dectin-1, does not affect

317 the course of murine experimental colitis, another group found Dectin-1 deficiency increased

318 susceptibility to colitis $(16,17)$. Hence, although BGs from different sources can vary structurally

319 and functionally (1), the true impact of orally administering BGs on gut inflammation and the

320 associated mechanisms is unknown, and the previously reported outcomes are non-conclusive 
321 without the use of well-defined, high pure, BGs such as the YBG used in this study. Here, we show

322 that prolonged oral administration of high pure YBG prior to DSS treatment to induce colitis

323 results in significantly lowered severity of gut inflammation in B6 mice. This diminished

324 susceptibility to DSS colitis is associated altered gut microbiota composition and function favoring

325 polysaccharide metabolism, higher immune regulatory SCFA production, and enhanced gut

326 immune regulation.

327 Intriguingly, we found that treatment with high pure YBG at disease induction stage alone has no

328 beneficial effects, but only prolonged oral pretreatment with this agent suppresses colitis

329 susceptibility. This shows that well defined BGs can promote protection from colitis, but it

330 depends on gradually acquired enhanced immune regulation, perhaps mediated by altered / YBG

331 shaped gut microbiota. In fact, although the role of immune regulation promoted directly by YBG

332 in the gut mucosa cannot be ruled out, we found that prolonged oral treatment using YBG causes

333 changes in the gut microbial composition. YBG consumption resulted in reduced abundance of

334 Firmicutes and increased abundance of Bacteroidetes and Verrucomicrobia phyla, which include

335 many polysaccharide fermenting bacteria $(49,50)$. However, YBG treatment also diminished gut

336 microbial diversity / species richness, suggesting that YBG treatment induced effect is due to

337 selective enrichment of microbial communities that promote immune regulation, but not by

338 increasing the diversity of gut microbiota. Further, predictive functional profiling of fecal

339 microbiota showed overrepresentation of metabolic pathways linked to carbohydrate metabolism,

340 glycan biosynthesis and metabolism, and biosynthesis of secondary metabolites after YBG

341 treatment compared to pre-treatment time-point. More importantly, our novel observations that

342 fecal levels of immune regulatory microbial SCFA $(51,52)$ such as acetic acid, propionic acid

343 and butyric acid produced by gut microbiota are higher in YBG treated mice suggest that altered

344 microbiota is functionally superior in terms of their ability to promote immune regulation. This 
345 notion has been validated by diminishes levels of these SCFAs, Treg frequencies in the gut

346 mucosa, and elimination of YBG treatment induced effect upon depletion of gut microbiota.

347 Further, the need for prolonged pre-treatment, which could potentially help gradual shaping of gut

348 microbiota structure and function, for protection from colitis is another indication that altered gut

349 microbiota is the key contributor of protection from colitis in YBG fed mice.

350 Colonic microbial communities can ferment non-digestible dietary polysaccharides to use them as

351 energy sources for their growth, and also produce small metabolites such as SCFA that enhance

352 gut immune regulation $(16,49,51,53-58)$. Previous studies have shown that gut colonization by

353 specific commensal microbial communities causes induction and expansion of Foxp3+ Tregs in

354 the intestinal and systemic compartments (59-61). Further, gut bacteria play a critical role in

355 maintaining peripheral tolerance and gut immune homeostasis $(62,63)$. However, it is not known

356 if BG shaped gut microbes promote Treg induction and/or expansion. Our observations that YBG

357 treatment increases the production of microbial SCFA, which are known to enhance gut integrity

358 and immune regulation $(51,55)$, suggest that microbiota does contribute to YBG treatment

359 associated increase in overall immune regulation. Importantly, abundances of microbes belonging

360 to polysaccharide degrading and well as host beneficial communities such as Parabacteroides,

361 Sutterella, Prevotella, Bifidobacterium and Akkermansia are increased upon prolonged YBG treatment.

362 Moreover, microbial depletion and reduced SCFA production resulted in increased production of

363 pro-inflammatory cytokines IFN $\gamma$ and IL17 in the distal gut of YBG treated mice substantiating

364 the notion that microbes contributes to enhance immune regulation and diminished susceptibility

365 to colitis when pre-treated with this CDP.

366 Although this study is focused on the impact of YBG treatment on colitis susceptibility and distal

367 gut, we found that, unlike the large intestine, small intestine of YBG treated mice, with intact and 
368 depleted microbiota, have relatively higher frequencies of Tregs compared to untreated controls.

369 This suggests that YBG-treatment associated increase in Treg abundance in small and large

370 intestinal compartments may involve different mechanisms. It is possible that while prolonged

371 treatment with YBG, at the employed human relevant dose, interacts directly with small intestinal

372 mucosa to enhance immune regulation, YBG treatment associated enhanced immune regulation in

373 the large intestine may be primarily microbiota (and SCFA) dependent. Of note, our recent report

374 showed that treatment of mice with a higher dose of this YBG caused strong pro-inflammatory

375 immune response in both small and large intestine (29), suggesting that use of higher doses of this

376 CDP is not advisable to ameliorate susceptibility to gut inflammation.

377 Although we previously reported that Dectin-1 dependent interaction of high pure YBG with

378 immune cells could promote a combination of both pro-inflammatory and immune regulatory

379 immune responses $(19,27)$ under normal circumstances, our current study suggest that such direct

380 interaction can produce primarily pathogenic response under depleted microbiota and pro-

381 inflammatory conditions such as a chemical insult using DSS. In fact it has been shown that the

382 degree and type of immune cell response to BGs depends on the cytokine milieu and

383 microenvironment (64). It is possible that YBG is degraded by colonic bacteria and produce

384 immune regulatory SCFA along with minimizing its direct interaction with large intestinal mucosa.

385 On the other hand, microbial depletion could diminish YBG degradation and SCFA production.

386 Further, DSS which causes epithelial damage can expose immune cells for gut mucosa for direct

387 interaction with YBG resulting in dominant pro-inflammation immune response. This is evident

388 from our observation that YBG treated, microbiota depleted mice presents more severe colitis

389 compared to YBG treated mice with intact microbiota, and control groups of mice with intact and

390 depleted microbiota. 
391 Overall, our studies using YBG found that this CDP can promote gut immune regulation by

392 altering gut microbiota, increasing SCFA production, enhancing the overall immune regulation

393 and gut health under normal circumstances suggesting the prebiotic value of BGs. Importantly,

394 only pre-conditioning of the intestinal microenvironment in terms of altering gut microbiota

395 composition and function, which enhances SCFA production and immune regulation, by treating

396 with YBG diminishes susceptibility to chemical induced colitis. We show that YBG treatment has

397 no benefits in ameliorating the severity of ongoing gut inflammation, as in the case of UC. Hence,

398 with respect to the colitis, health benefits of YBG could be limited to those who are susceptible to,

399 but not yet developed, the disease rather than those who have established disease. We employed

400 microbiota depletion approach in our study primarily to understand the mechanism by which YBG

401 treatment enhances gut immune regulation and diminish colitis susceptibility. Nevertheless, our

402 observations suggest that consumption of YBG, as a dietary supplement, by those who are under

403 antibiotic therapy could produce adverse effects such as gut inflammation. Of note, utility of

404 antibiotic treatments have been considered for ameliorating the severity of gut inflammation and

405 for managing UC symptoms $(65,66)$. Our observations suggest the possibility that consumption

406 of YBG as a dietary supplement by UC patients who are under such therapy will result in

407 aggravated, rather than ameliorated, disease severity. In conclusion, this study, which used high

408 pure YBG, not only demonstrates the dietary supplement value of BGs in promoting gut health

409 and the associated mechanisms, but also show that these CDPs may not be beneficial in

410 suppressing ongoing gut inflammation and they produce adverse effects when used in combination

411 with antibiotic therapy. 
412 Conflict of Interest statement: Authors do not have any conflict(s) of interest to disclose.

413 Author contribution: R.B., B.J, and J.S. researched and analyzed data, and R.G. researched and

414 analyzed the data and edited the manuscript; C.V. designed experiments, researched and analyzed

415 data, and wrote/reviewed/edited manuscript.

416

417 Acknowledgments: This work was supported by internal funds from MUSC, National Institutes

418 of Health (NIH) grants: R21AI133798 (NIAID), and R21AI133798 and R21AI136339-02S1

419 Administrate supplements (Office of Dietary Supplements) to CV. The authors are thankful to the

420 Histology core of Pathology department of MUSC for the histology service, and Genomic center

421 of MUSC for 16S rRNA gene sequencing service. Dr. Vasu is the guarantor of this work and as

422 such, has full access to all the data in the study and takes responsibility for integrity of the data

423 and accuracy of data analysis. 
426 FIGURE 1: Effect of pre-treatment with YBG (A) on induced colitis associated weight loss (B),

427 colon shortening (C) and colon inflammation (D) in B6 mice. (A) Cartoon depicting experimental

428 design is shown. (B) Body weights of individual animals were measured every day starting at day

42930 and changes in percentage of body weights, relative to initial body weight, are shown. (C)

430 Images of representative colons (left panel) and colon lengths (right panel) of euthanized mice are

431 shown. (D) H\&E stained distal colon sections were evaluated for inflammation and images of 432 representative sections (left panel) and inflammation severity scores (right panel) are shown. (B, 433 right panels of C, D) Mean \pm SDs $(n=8) . *{ }^{*}, * * *$ Different from control, $P<0.05, P<0.01, P<0.001$ 434 respectively.

435 FIGURE 2: Effect of YBG pre-treatment on Treg (A) and cytokine expressing (B) CD4+ T

436 frequencies in, and cytokine secretion (C) by, MLN cells of B6 mice with DSS induced colitis.

$437(\mathrm{~A}, \mathrm{~B})$ Frequencies of CD4+ cells in the MLN single cell suspension that express indicated factor 438 determined by FACS are shown. C) Concentrations of indicated cytokines secreted by MLN cells 439 upon ex vivo stimulation using anti-CD3 antibody are shown. Mean \pm SDs $(n=5)$ are shown for all 440 panels. *** Different from control, $P<0.05, P<0.01$ respectively.

441 FIGURE 3: Impact of oral administration of YBG on the composition of gut microbiota at genus

442 level (A) and predictive functions (B) in B6 mice. (A) OTU data of 16S rRNA gene sequences of

443 fecal pellet collected before (day 0) and 30 days after YBG treatment (day 30) were compiled to

444 genus level based upon the percentage identity to reference sequences (i.e. $>97 \%$ identity) and

445 heatmap was generated using sequence counts of major genera (those with $>1 \%$ of total

446 sequences) that showed statistically significant differences. (B) OUT-biom data was used for

447 predicting gene functional categories using PICRUSt application and the level 2 categories of 
448 KEGG pathway that showed statistically significant differences are shown. $n=5$ for both panels.

$449 *, * *, * * *$ Different from control, $P<0.05, P<0.01, P<0.001$ respectively.

450 FIGURE 4: Impact of oral administration of YBG on the Treg frequency and cytokine secretion

451 profile of immune cells in B6 mice with intact (A) and depleted (B) gut microbiota. (A, B) Foxp3+

452 CD4 cell frequencies in gut associated lymphoid tissues (MLN, SiLP and LiLP) determined by

453 FACS (left panels) and the levels of indicated cytokines secreted by MLN upon stimulation using

454 anti-CD3 antibody determined by multiplex assay (right panels) are shown. Mean \pm SDs $(n=5)$ are

455 shown for all panels. *,** Different from control, $P<0.05, P<0.01$ respectively.

456 FIGURE 5: Impact of oral administration of YBG on the fecal SCFA levels in B6 mice with intact

457 (A) and depleted (B) gut microbiota. (A, B) SCFA levels in fecal pellets collected from control 458 and YBG treated mice that were left alone or given broad spectrum antibiotics are shown.

459 Mean \pm SDs $(n=5)$ are shown for all panels. *Different from control, $P<0.05$.

460 FIGURE 6: Effect of microbiota depletion (A) in DSS colitis induced weight loss (B), colon 461 inflammation (C) and cytokine secretion by intestinal immune cells (D) in control and YBG treated 462 B6 mice. (A) Cartoon depicting experimental design is shown. (B) Body weights of individual 463 animals were measured every day starting at day 30 and changes in percentage of body weights, 464 relative to initial body weight, are shown. (C) H\&E stained distal colon sections were evaluated 465 for inflammation and the severity scores are shown. D) Concentrations of indicated cytokines 466 secreted by colonic immune cells upon ex vivo stimulation using anti-CD3 antibody are shown.

467 (B,C, D) Mean \pm SDs $(n=5)$ are shown. *, **,*** Different from control, $P<0.05, P<0.01, P<0.001$ 468 respectively. 


\section{References:}

1. Synytsya A, Novak M. Structural analysis of glucans. Ann Transl Med. 2014 Feb;2:17.

2. Chen J, Seviour R. Medicinal importance of fungal beta-(1-->3), (1-->6)-glucans. Mycological research. 2007 Jun;111:635-52.

3. Vannucci L, Krizan J, Sima P, Stakheev D, Caja F, Rajsiglova L, Horak V, Saieh M. Immunostimulatory properties and antitumor activities of glucans (Review). International journal of oncology. 2013 Aug;43:357-64.

4. El Khoury D, Cuda C, Luhovyy BL, Anderson GH. Beta glucan: health benefits in obesity and metabolic syndrome. J Nutr Metab. 2012;2012:851362.

5. Mosikanon K, Arthan D, Kettawan A, Tungtrongchitr R, Prangthip P. Yeast beta-Glucan Modulates Inflammation and Waist Circumference in Overweight and Obese Subjects. J Diet Suppl. 2017 Mar 4;14:173-85.

6. Zou Y, Liao D, Huang H, Li T, Chi H. A systematic review and meta-analysis of betaglucan consumption on glycemic control in hypercholesterolemic individuals. Int J Food Sci Nutr. 2015;66:355-62.

7. Fuller R, Moore MV, Lewith G, Stuart BL, Ormiston RV, Fisk HL, Noakes PS, Calder PC. Yeast-derived beta-1,3/1,6 glucan, upper respiratory tract infection and innate immunity in older adults. Nutrition. 2017 Jul - Aug;39-40:30-5.

8. McFarlin BK, Carpenter KC, Davidson T, McFarlin MA. Baker's yeast beta glucan supplementation increases salivary IgA and decreases cold/flu symptomatic days after intense exercise. J Diet Suppl. 2013 Sep;10:171-83.

9. Leentjens J, Quintin J, Gerretsen J, Kox M, Pickkers P, Netea MG. The effects of orally administered Beta-glucan on innate immune responses in humans, a randomized open-label intervention pilot-study. PloS one. 2014;9:e108794.

10. Kamiya T, Tang C, Kadoki M, Oshima K, Hattori M, Saijo S, Adachi Y, Ohno N, Iwakura Y. beta-Glucans in food modify colonic microflora by inducing antimicrobial protein, calprotectin, in a Dectin-1-induced-IL-17F-dependent manner. Mucosal Immunol. 2018 May;11:763-73.

11. Liu B, Lin Q, Yang T, Zeng L, Shi L, Chen Y, Luo F. Oat beta-glucan ameliorates dextran sulfate sodium (DSS)-induced ulcerative colitis in mice. Food Funct. 2015 Nov;6:3454-63.

12. Chung MY, Hwang JT, Kim JH, Shon DH, Kim HK. Sarcodon aspratus Extract Ameliorates Dextran Sulfate Sodium-Induced Colitis in Mouse Colon and Mesenteric Lymph Nodes. J Food Sci. 2016 May;81:H1301-8.

13. Sun Y, Shi X, Zheng X, Nie S, Xu X. Inhibition of dextran sodium sulfate-induced colitis in mice by baker's yeast polysaccharides. Carbohydr Polym. 2019 Mar 1;207:371-81.

14. Zhou M, Wang Z, Chen J, Zhan Y, Wang T, Xia L, Wang S, Hua Z, Zhang J. Supplementation of the diet with Salecan attenuates the symptoms of colitis induced by dextran sulphate sodium in mice. Br J Nutr. 2014 May 28;111:1822-9.

15. Heinsbroek SE, Williams DL, Welting O, Meijer SL, Gordon S, de Jonge WJ. Orally delivered beta-glucans aggravate dextran sulfate sodium (DSS)-induced intestinal inflammation. Nutr Res. 2015 Dec;35:1106-12.

16. Heinsbroek SE, Oei A, Roelofs JJ, Dhawan S, te Velde A, Gordon S, de Jonge WJ. Genetic deletion of dectin-1 does not affect the course of murine experimental colitis. BMC Gastroenterol. 2012 Apr 16;12:33.

17. Iliev ID, Funari VA, Taylor KD, Nguyen Q, Reyes CN, Strom SP, Brown J, Becker CA, Fleshner PR, et al. Interactions between commensal fungi and the C-type lectin receptor Dectin-1 influence colitis. Science. 2012 Jun 8;336:1314-7. 
18. Bacon JS, Farmer VC, Jones D, Taylor IF. The glucan components of the cell wall of baker's yeast (Saccharomyces cerevisiae) considered in relation to its ultrastructure. The Biochemical journal. 1969 Sep;114:557-67.

19. Gudi R, Perez N, Johnson BM, Sofi MH, Brown R, Quan S, Karumuthil-Melethil S, Vasu

C. Complex dietary polysaccharide modulates gut immune function and microbiota, and promotes protection from autoimmune diabetes. Immunology. 2019 Feb 3. Pierre P, et al. Low-Molecular-Weight Peptides from Salmon Protein Prevent Obesity-Linked Glucose Intolerance, Inflammation, and Dyslipidemia in LDLR-/-/ApoB100/100 Mice. J Nutr. 2015 Jul;145:1415-22.

21. Mahler M, Bristol IJ, Leiter EH, Workman AE, Birkenmeier EH, Elson CO, Sundberg JP. Differential susceptibility of inbred mouse strains to dextran sulfate sodium-induced colitis. Am J Physiol. 1998 Mar;274:G544-51.

22. Taghipour N, Molaei M, Mosaffa N, Rostami-Nejad M, Asadzadeh Aghdaei H, Anissian A, Azimzadeh P, Zali MR. An experimental model of colitis induced by dextran sulfate sodium from acute progresses to chronicity in $\mathrm{C} 57 \mathrm{BL} / 6$ : correlation between conditions of mice and the environment. Gastroenterol Hepatol Bed Bench. 2016 Winter;9:45-52.

23. Eichele DD, Kharbanda KK. Dextran sodium sulfate colitis murine model: An indispensable tool for advancing our understanding of inflammatory bowel diseases pathogenesis. World J Gastroenterol. 2017 Sep 7;23:6016-29.

24. DeVoss J, Diehl L. Murine models of inflammatory bowel disease (IBD): challenges of modeling human disease. Toxicol Pathol. 2014 Jan;42:99-110. 25. Dennehy KM, Ferwerda G, Faro-Trindade I, Pyz E, Willment JA, Taylor PR, Kerrigan A, Tsoni SV, Gordon S, et al. Syk kinase is required for collaborative cytokine production induced through Dectin-1 and Toll-like receptors. European journal of immunology. 2008 Feb;38:500-6. 26. Dennehy KM, Willment JA, Williams DL, Brown GD. Reciprocal regulation of IL-23 and IL-12 following co-activation of Dectin-1 and TLR signaling pathways. European journal of immunology. 2009 May;39:1379-86.

27. Karumuthil-Melethil S, Gudi R, Johnson BM, Perez N, Vasu C. Fungal beta-glucan, a Dectin-1 ligand, promotes protection from type 1 diabetes by inducing regulatory innate immune response. J Immunol. 2014 Oct 1;193:3308-21.

28. Montufar-Solis D, Klein JR. An improved method for isolating intraepithelial lymphocytes (IELs) from the murine small intestine with consistently high purity. J Immunol Methods. 2006 Jan 20;308:251-4.

29. Gudi R, Perez N, Johnson BM, Sofi MH, Brown R, Quan S, Karumuthil-Melethil S, Vasu C. Complex dietary polysaccharide modulates gut immune function and microbiota, and promotes protection from autoimmune diabetes. Immunology. 2019 May; 157:70-85.

30. Sofi MH, Johnson BM, Gudi RR, Jolly A, Gaudreau MC, Vasu C. Polysaccharide ADependent Opposing Effects of Mucosal and Systemic Exposures to Human Gut Commensal Bacteroides fragilis in Type 1 Diabetes. Diabetes. 2019 Oct;68:1975-89.

31. Chassaing B, Aitken JD, Malleshappa M, Vijay-Kumar M. Dextran sulfate sodium (DSS)induced colitis in mice. Curr Protoc Immunol. 2014 Feb 4;104:Unit 1525.

32. Erben U, Loddenkemper C, Doerfel K, Spieckermann S, Haller D, Heimesaat MM, Zeitz M, Siegmund B, Kuhl AA. A guide to histomorphological evaluation of intestinal inflammation in mouse models. Int J Clin Exp Pathol. 2014;7:4557-76.

559 33. Gudi RR, Karumuthil-Melethil S, Perez N, Li G, Vasu C. Engineered Dendritic Cell560 Directed Concurrent Activation of Multiple T cell Inhibitory Pathways Induces Robust Immune 561 Tolerance. Sci Rep. 2019 Aug 19;9:12065. 
562

563

564

565

566

567

568

569

570

571

572

573

574

575

576

577

578

579

580

581

582

583

584

585

586

587

588

589

590

591

592

593

594

595

596

597

598

599

600

601

602

603

604

605

606

607

608

34. Johnson BM, Gaudreau MC, Al-Gadban MM, Gudi R, Vasu C. Impact of dietary deviation on disease progression and gut microbiome composition in lupus-prone SNF1 mice. Clin Exp Immunol. 2015 Aug;181:323-37.

35. Sofi MH, Gudi R, Karumuthil-Melethil S, Perez N, Johnson BM, Vasu C. pH of drinking water influences the composition of gut microbiome and type 1 diabetes incidence. Diabetes. 2014 Feb;63:632-44.

36. Caporaso JG, Kuczynski J, Stombaugh J, Bittinger K, Bushman FD, Costello EK, Fierer $\mathrm{N}$, Pena AG, Goodrich JK, et al. QIIME allows analysis of high-throughput community sequencing data. Nature methods. 2010 May;7:335-6.

37. Wixon J, Kell D. The Kyoto encyclopedia of genes and genomes--KEGG. Yeast. 2000 Apr;17:48-55.

38. Parks DH, Beiko RG. Identifying biologically relevant differences between metagenomic communities. Bioinformatics. 2010 Mar 15;26:715-21.

39. Hevia A, Milani C, Lopez P, Cuervo A, Arboleya S, Duranti S, Turroni F, Gonzalez S, Suarez A, et al. Intestinal dysbiosis associated with systemic lupus erythematosus. mBio. 2014;5:e01548-14.

40. Zhang H, Liao X, Sparks JB, Luo XM. Dynamics of gut microbiota in autoimmune lupus. Applied and environmental microbiology. 2014 Dec 15;80:7551-60.

41. Parks DH, Tyson GW, Hugenholtz P, Beiko RG. STAMP: statistical analysis of taxonomic and functional profiles. Bioinformatics. 2014 Nov 1;30:3123-4.

42. Dhariwal A, Chong J, Habib S, King IL, Agellon LB, Xia J. MicrobiomeAnalyst: a webbased tool for comprehensive statistical, visual and meta-analysis of microbiome data. Nucleic Acids Res. 2017 Jul 3;45:W180-W8.

43. Zhao G, Nyman M, Jonsson JA. Rapid determination of short-chain fatty acids in colonic contents and faeces of humans and rats by acidified water-extraction and direct-injection gas chromatography. Biomed Chromatogr. 2006 Aug;20:674-82.

44. Ramberg JE, Nelson ED, Sinnott RA. Immunomodulatory dietary polysaccharides: a systematic review of the literature. Nutr J. 2010 Nov 18;9:54.

45. Hong F, Yan J, Baran JT, Allendorf DJ, Hansen RD, Ostroff GR, Xing PX, Cheung NK, Ross GD. Mechanism by which orally administered beta-1,3-glucans enhance the tumoricidal activity of antitumor monoclonal antibodies in murine tumor models. Journal of immunology. 2004 Jul 15;173:797-806.

46. Suzuki I, Tanaka H, Kinoshita A, Oikawa S, Osawa M, Yadomae T. Effect of orally administered beta-glucan on macrophage function in mice. Int J Immunopharmacol. 1990;12:67584.

47. Suzuki I, Hashimoto K, Ohno N, Tanaka H, Yadomae T. Immunomodulation by orally administered beta-glucan in mice. Int J Immunopharmacol. 1989;11:761-9.

48. Volman JJ, Ramakers JD, Plat J. Dietary modulation of immune function by beta-glucans. Physiol Behav. 2008 May 23;94:276-84.

49. Flint HJ, Scott KP, Duncan SH, Louis P, Forano E. Microbial degradation of complex carbohydrates in the gut. Gut Microbes. 2012 Jul-Aug;3:289-306.

50. Martinez-Garcia M, Brazel DM, Swan BK, Arnosti C, Chain PS, Reitenga KG, Xie G, Poulton NJ, Lluesma Gomez M, et al. Capturing single cell genomes of active polysaccharide degraders: an unexpected contribution of Verrucomicrobia. PloS one. 2012;7:e35314.

51. Marino E, Richards JL, McLeod KH, Stanley D, Yap YA, Knight J, McKenzie C, Kranich $\mathrm{J}$, Oliveira AC, et al. Gut microbial metabolites limit the frequency of autoimmune T cells and protect against type 1 diabetes. Nature immunology. 2017 May;18:552-62. 
52. D'Souza WN, Douangpanya J, Mu S, Jaeckel P, Zhang M, Maxwell JR, Rottman JB, Labitzke K, Willee A, et al. Differing roles for short chain fatty acids and GPR43 agonism in the regulation of intestinal barrier function and immune responses. PloS one. 2017;12:e0180190.

612 53. Porter NT, Martens EC. The Critical Roles of Polysaccharides in Gut Microbial Ecology and Physiology. Annu Rev Microbiol. 2017 Sep 8;71:349-69.

614 54. Temple MJ, Cuskin F, Basle A, Hickey N, Speciale G, Williams SJ, Gilbert HJ, Lowe EC. A Bacteroidetes locus dedicated to fungal 1,6-beta-glucan degradation: Unique substrate conformation drives specificity of the key endo-1,6-beta-glucanase. The Journal of biological chemistry. 2017 Jun 23;292:10639-50.

618 55. Geirnaert A, Calatayud M, Grootaert C, Laukens D, Devriese S, Smagghe G, De Vos M, Boon N, Van de Wiele T. Butyrate-producing bacteria supplemented in vitro to Crohn's disease patient microbiota increased butyrate production and enhanced intestinal epithelial barrier integrity. Sci Rep. 2017 Sep 13;7:11450. 56. Turunen K, Tsouvelakidou E, Nomikos T, Mountzouris KC, Karamanolis D, Triantafillidis J, Kyriacou A. Impact of beta-glucan on the faecal microbiota of polypectomized patients: a pilot study. Anaerobe. 2011 Dec;17:403-6. Health: Comparing the in Vitro Fermentations of Beta-Glucan, Inulin and Xylooligosaccharide. Nutrients. 2017 Dec 15;9.

628 58. Holscher HD. Dietary fiber and prebiotics and the gastrointestinal microbiota. Gut Microbes. 2017 Mar 4;8:172-84.

630 59. Furusawa Y, Obata Y, Fukuda S, Endo TA, Nakato G, Takahashi D, Nakanishi Y, Uetake $\mathrm{C}$, Kato $\mathrm{K}$, et al. Commensal microbe-derived butyrate induces the differentiation of colonic regulatory T cells. Nature. 2013 Dec 19;504:446-50.

60. Ye J, Qiu J, Bostick JW, Ueda A, Schjerven H, Li S, Jobin C, Chen ZE, Zhou L. The Aryl Hydrocarbon Receptor Preferentially Marks and Promotes Gut Regulatory T Cells. Cell Rep. 2017 Nov 21;21:2277-90.

636 61. Chang PV, Hao L, Offermanns S, Medzhitov R. The microbial metabolite butyrate regulates intestinal macrophage function via histone deacetylase inhibition. Proceedings of the National Academy of Sciences of the United States of America. 2014 Feb 11;111:2247-52.

$640 \quad 18 ; 46: 562-76$.

641 63. Steinmeyer S, Lee K, Jayaraman A, Alaniz RC. Microbiota metabolite regulation of host 642 immune homeostasis: a mechanistic missing link. Curr Allergy Asthma Rep. 2015 May;15:24.

643 64. Municio C, Alvarez Y, Montero O, Hugo E, Rodriguez M, Domingo E, Alonso S, 644 Fernandez N, Crespo MS. The response of human macrophages to beta-glucans depends on the 645 inflammatory milieu. PloS one. 2013;8:e62016.

646 65. Nitzan O, Elias M, Peretz A, Saliba W. Role of antibiotics for treatment of inflammatory

648 66. Turner D, Levine A, Kolho KL, Shaoul R, Ledder O. Combination of oral antibiotics may 649 be effective in severe pediatric ulcerative colitis: a preliminary report. J Crohns Colitis. 2014 650 Nov;8:1464-70. 
Day (post DSS treatment initiation)

D

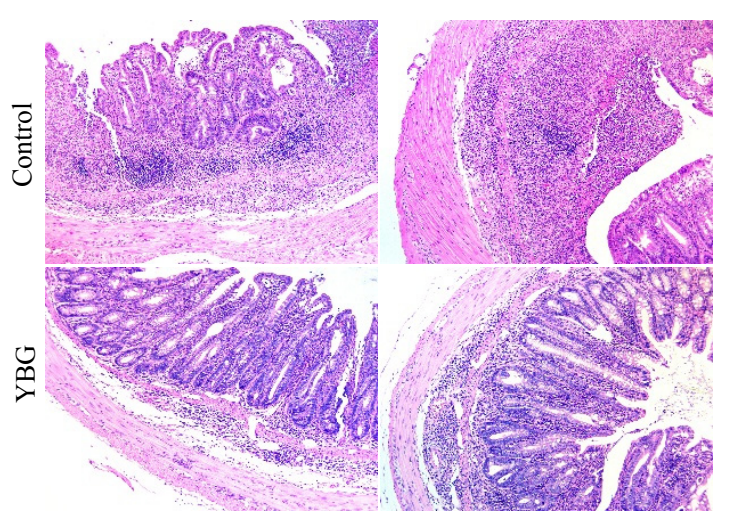

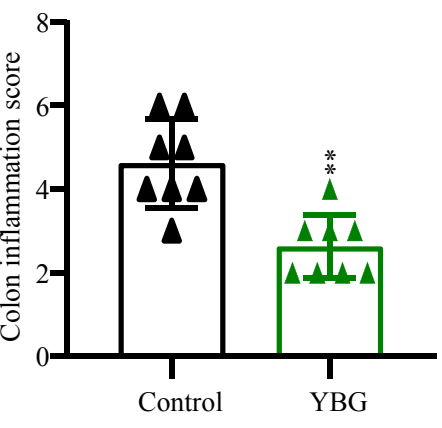

Fig. 1 bioRxiv preprint doi: https://doi.org/10.1101/719112; this version posted October 15, 2019. The copyright holder for this preprint (which was not
certified by peer review) is the author/funder. All rights reserved. No reuse allowed without permission.

Fig. 1 bioRxiv preprint doi: https://doi.org/10.1101/719112; this version posted October 15, 2019. The copyright holder for this preprint (which was not
certified by peer review) is the author/funder. All rights reserved. No reuse allowed without permission.

A

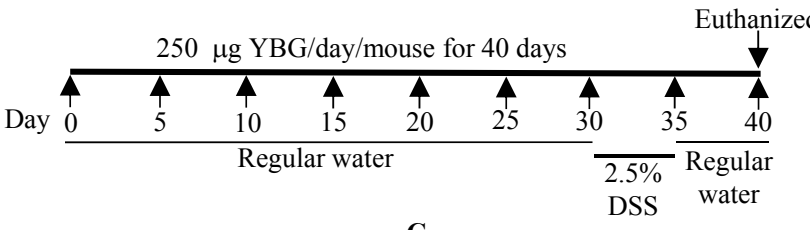

B

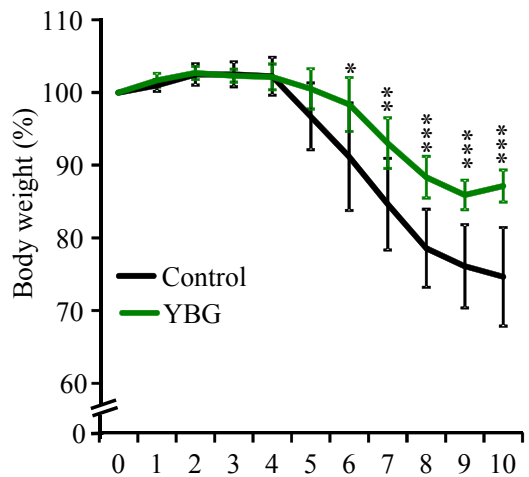

C

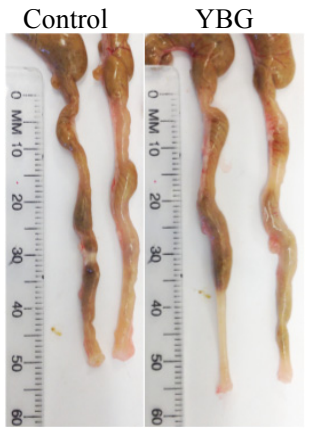

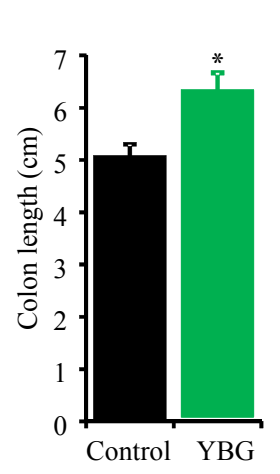


Fig. 2 bioRxiv preprint doi: https://doi.org/10.1101/719112; this version posted October 15,2019 . The copyright holder for this preprint (which was not
certified by peer review) is the author/funder. All rights reserved. No reuse allowed without permission.

Fig. 2 bioRxiv preprint doi: https://doi.org/10.1101/719112; this version posted October 15, 2019. The copyright holder for this preprint (which was not
certified by peer review) is the author/funder. All rights reserved. No reuse allowed without permission.

A

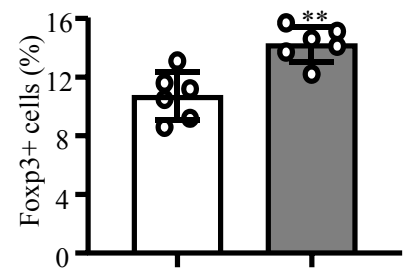

B
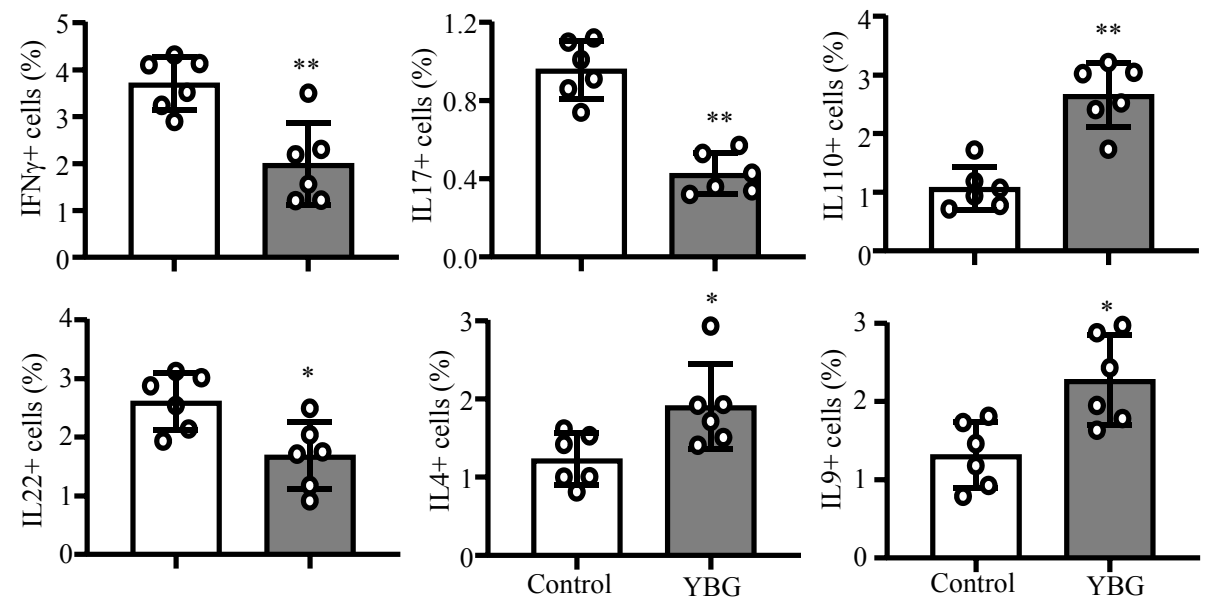

C
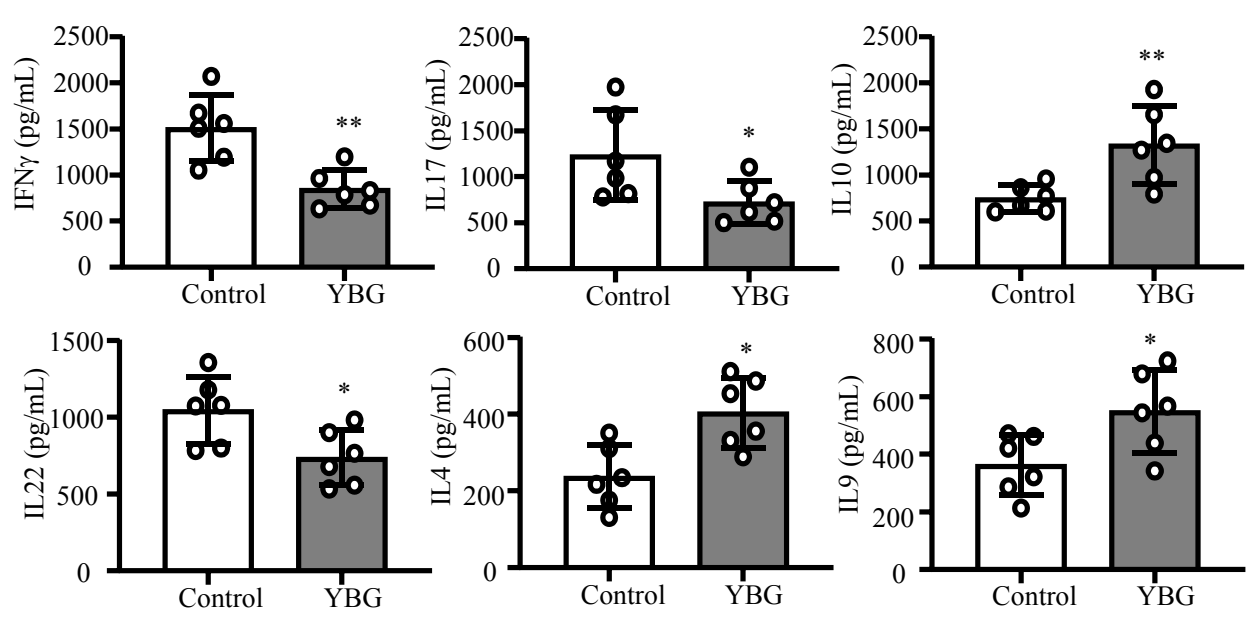

C 
Fig. 3 bioRxiv preprint doi: https://doi.org/10.1101/719112; this version posted October 15, 2019. The copyright holder for this preprint (which was not certified by peer review) is the author/funder. All rights reserved. No reuse allowed without permission.

A

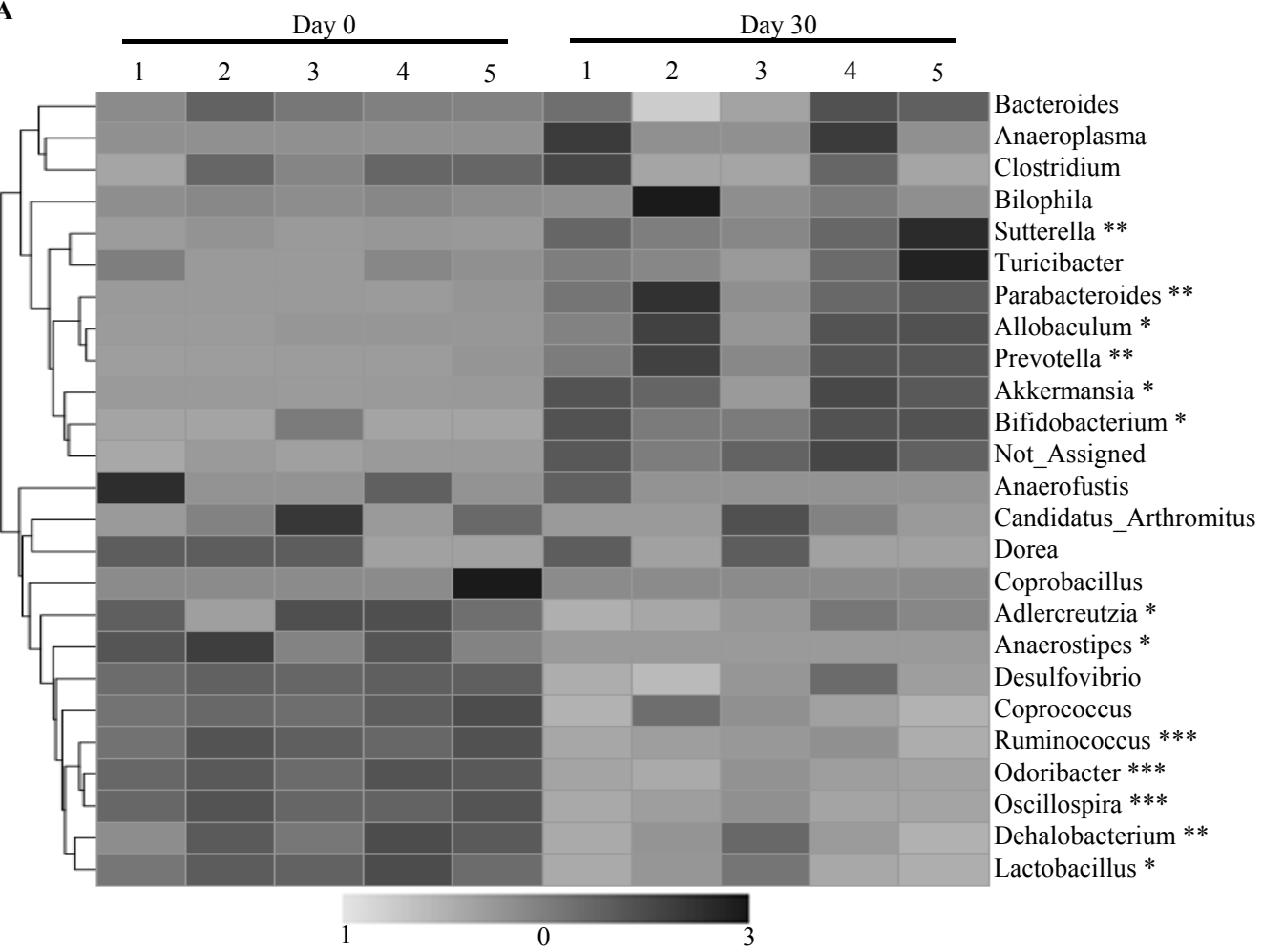

B

Day $0 \square$ Day 30

Metabolism of terpenoids and polyketides $\square * *$

Genetic information processing $\square * *$

Glycan biosynthesis and
metabolism

Carbohydrate metabolism

Biosynthesis of other
secondary metabolites

Energy metabolism Metabolism of cofactors
and vitamins

Nucleotide metabolism
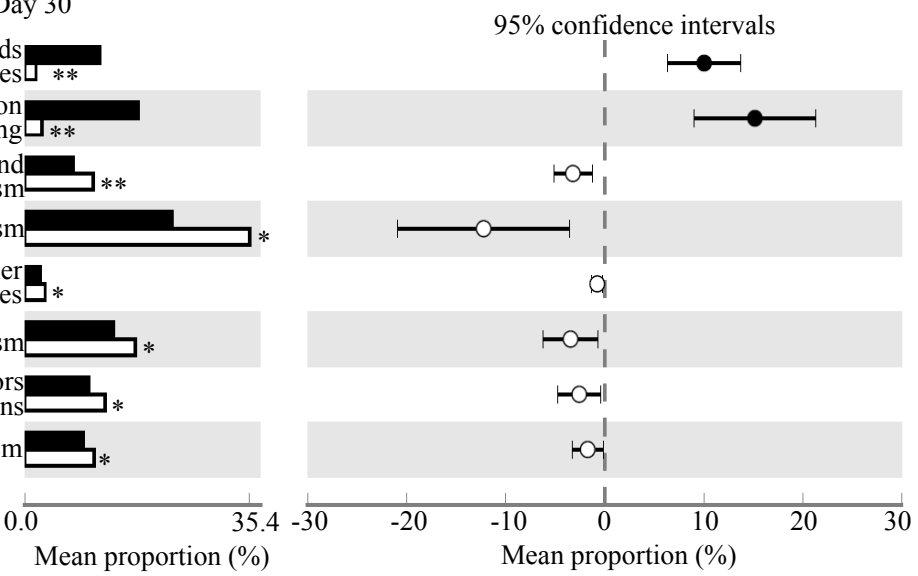
Fig. 4 bioRxiv preprint doi: https://doi.org/10.1101/719112; this version posted October 15, 2019. The copyright holder for this preprint (which was not certified by peer review) is the author/funder. All rights reserved. No reuse allowed without permission.

A

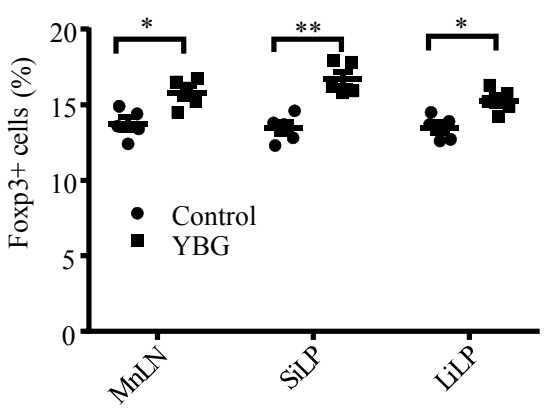

B

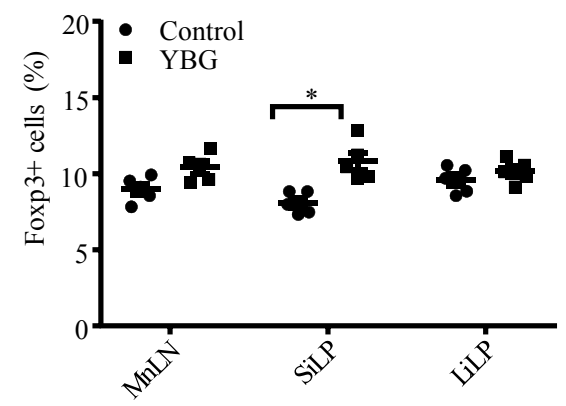

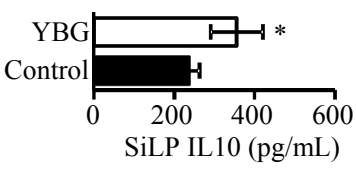
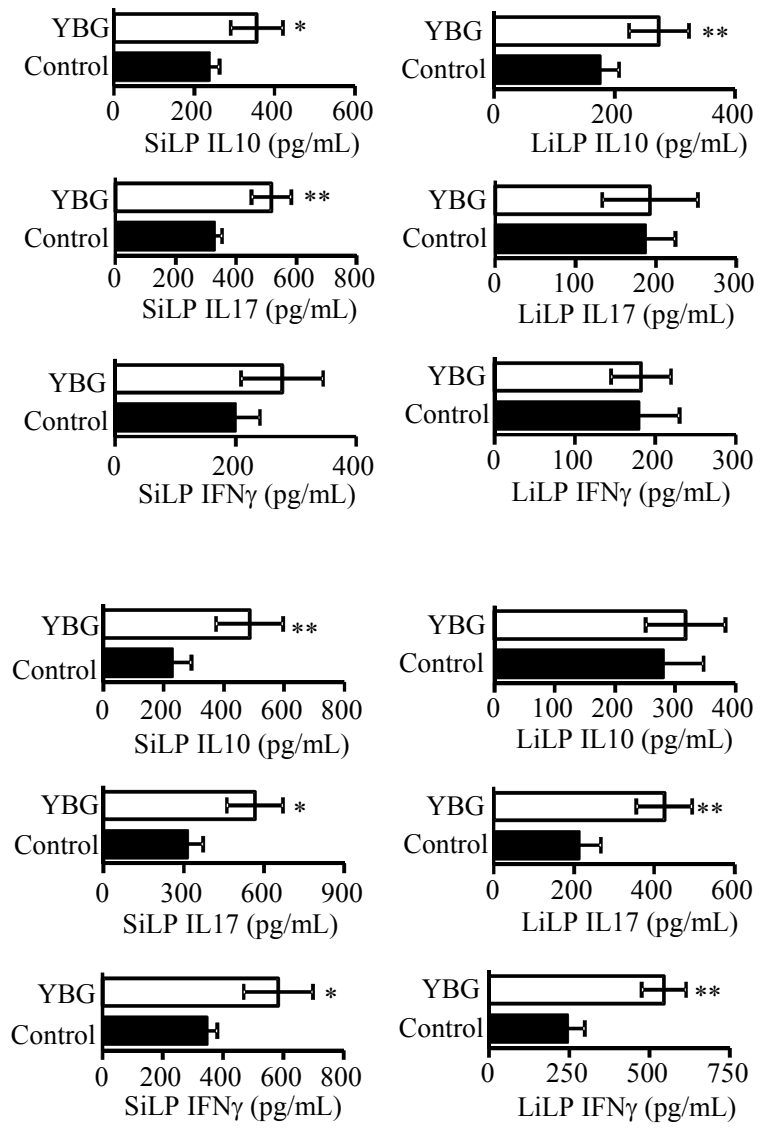

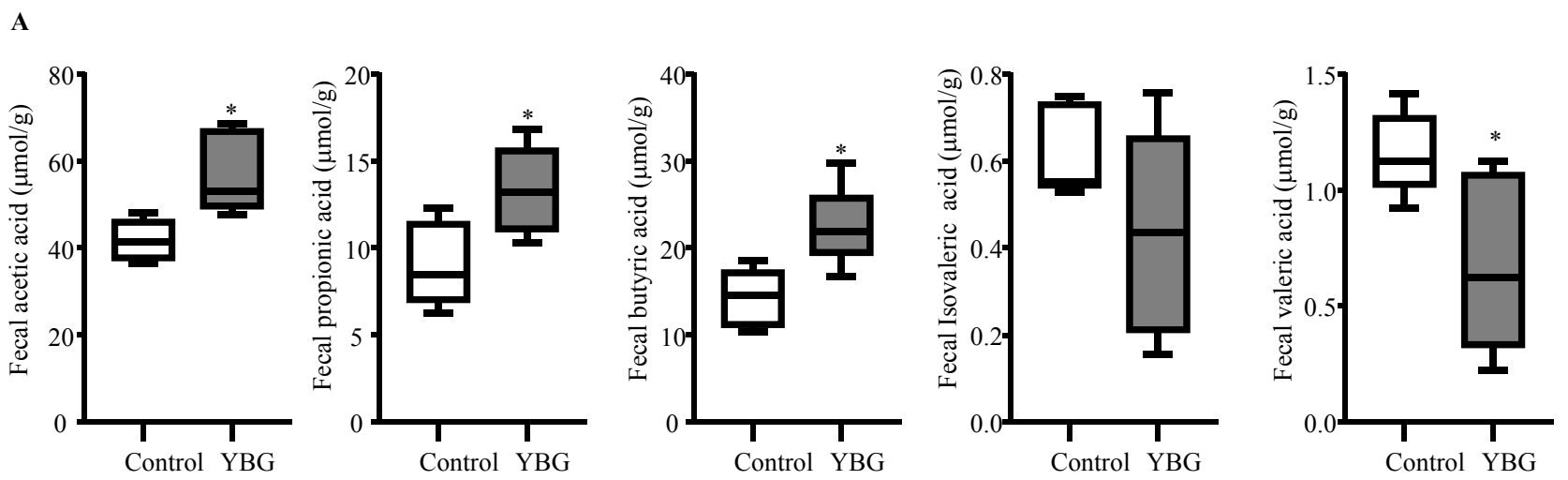

$\mathbf{B}$
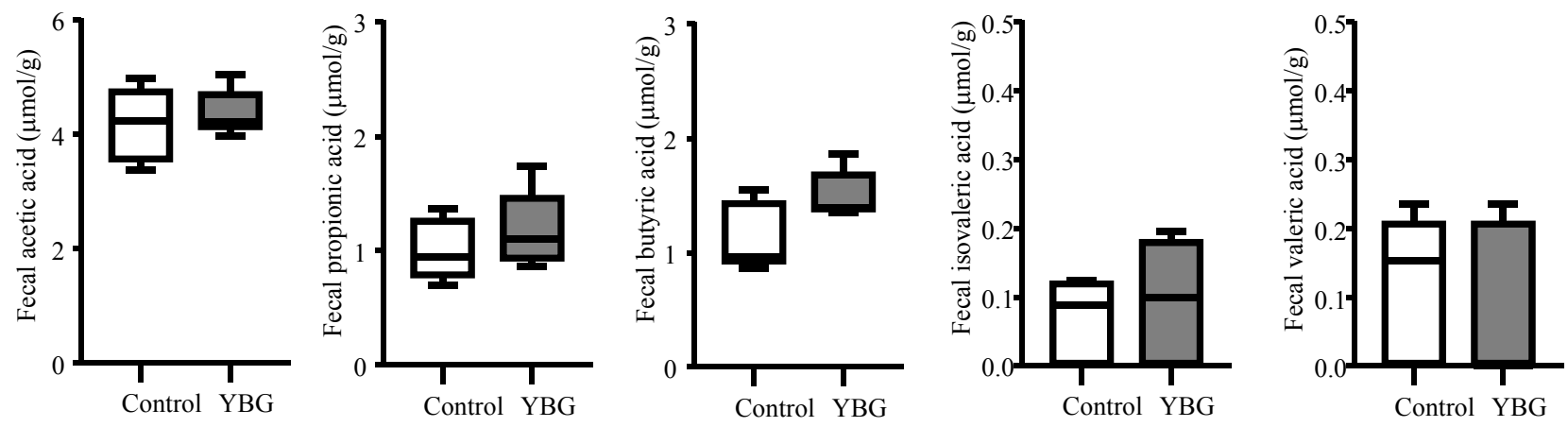

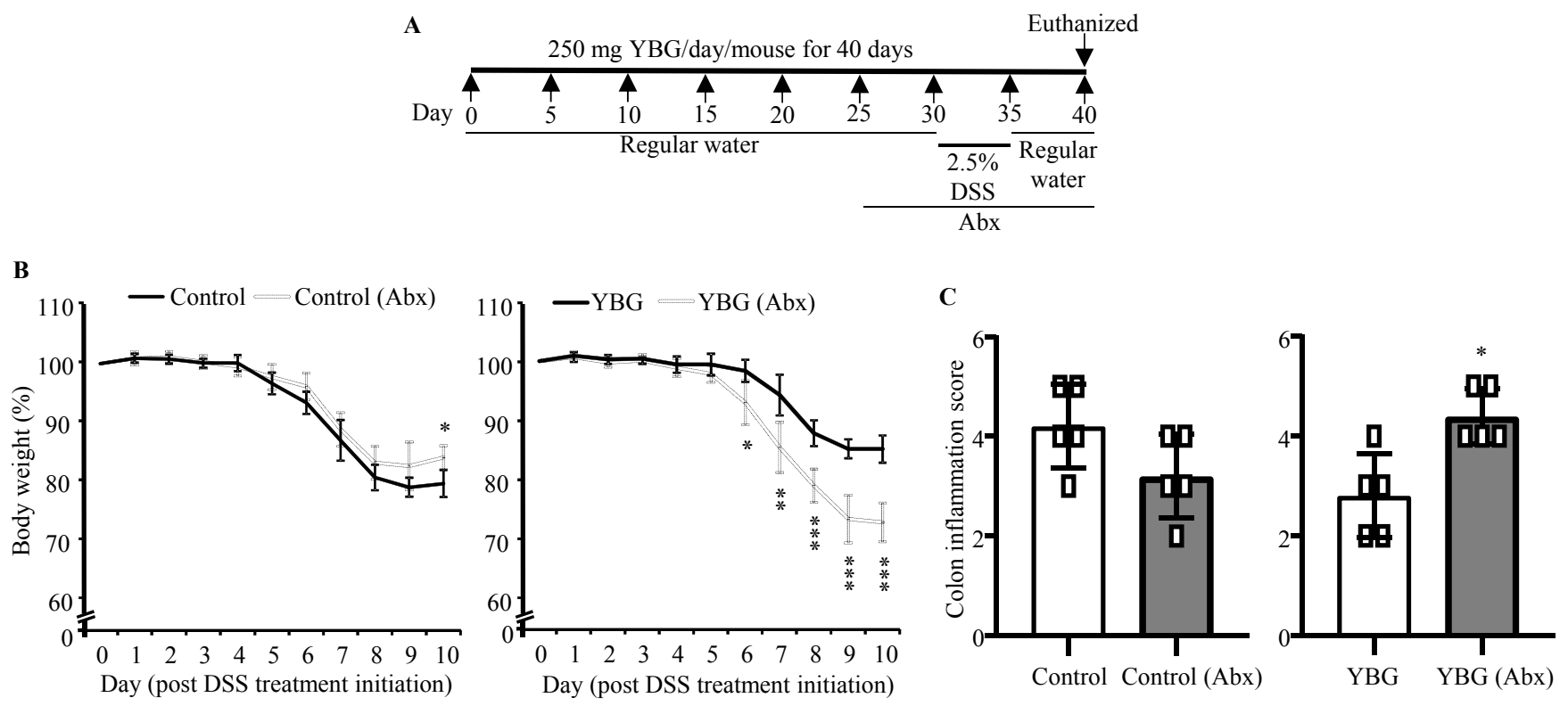

$$
\text { D }
$$

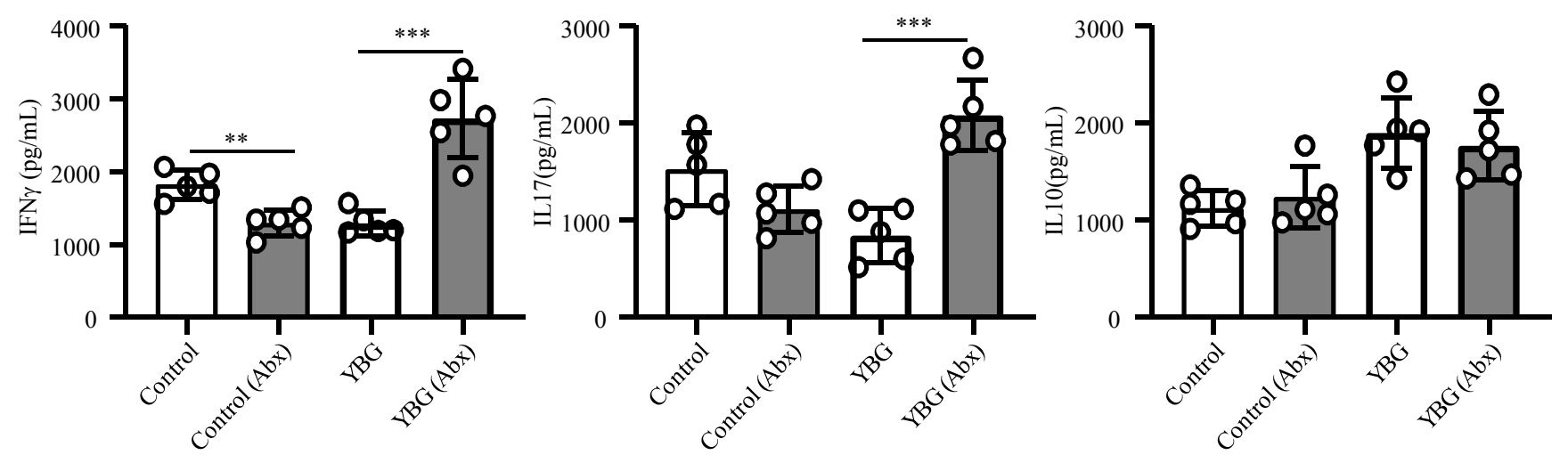

